Jakub Jasinski, Mathematics Department, University of Scranton, Scranton, PA 18510-2192, e-mail: jsj303@@tiger.uofs.edu

\title{
LARGE SETS CONTAINING COPIES OF SMALL SETS
}

\begin{abstract}
We investigate what large sets of real numbers such as uncountable, open, second Baire category, or positive Lebesgue measure contain copies of small sets such as finite, countable, or bounded. Some remarks on the related Erdös problem are included.
\end{abstract}

\section{Introduction, Sets with Copies of 3 Element Sets}

Let $X$ and $Y$ be sets of real numbers. We say that $X$ is similar to $Y$ if there exist real numbers $b$ and $c, c \neq 0$ such that

$$
c \cdot X+b \stackrel{d f}{=}\{c \cdot x+b: x \in X\}=Y .
$$

A set of real numbers $E$ contains a subset similar to the set $X$ (or copy of $X$ for short) if there exist real numbers $b$ and $c, c \neq 0$ such that $c \cdot X+b \subseteq E$. For example if $X$ is empty, then every set $E$ contains a copy of $X$. Obviously if $X$ consists of a single element then every nonempty set $E$ contains a copy of $X$. It is also clear that when $X$ consists of exactly two elements, then $E$ contains a copy of $X$ iff $\operatorname{card}(E) \geq 2$.

Before we discuss the case with $\operatorname{card}(X)=3$, we would like to point out that the concept of similarity is geometric in nature. On the other hand the sets $X$ and $E$ may originate from any field of mathematics. This gives the question, "Does $E$ contain a copy of $X$ ?" an interdisciplinary character. In this note we discuss the "classical" types of $X$ and $E$ which involve measure, the Baire property, and cardinality ${ }^{1}$.

\footnotetext{
Key Words: Lebesgue measure, Baire property, similar sets

Mathematical Reviews subject classification: Primary: 28A05 Secondary: 03E15, 28C10

Received by the editors June 21, 1995

${ }^{1}$ Most definitions and notation can be found in [8].
} 
Now let $X$ be a set with three elements. Arias de Reyna, using transfinite induction, proved [1] that there is a large (of the cardinality of the continuum, full outer Lebesgue measure, and not meager) set $E$ without a copy of $X$. Arias de Reyna's set is very irregular; it doesn't have the Baire property and is non-measurable. His result raises the question, "Does there exist a regular (i.e. measurable and/or with the Baire property) set of the cardinality of the continuum without a copy of a given three-element set?" A somewhat stronger result is stated below as Theorem 2. To prove it we need to notice that Theorem 2 of Mycielski [7] implies the following fact.

Theorem 1 If $\mathbb{K} \subset \mathbb{R}$ is a countable subfield, then there exists an uncountable closed set $E$ whose elements are linearly independent over $\mathbb{K}$.

Theorem 2 Let $\mathcal{X}$ be a countable family of three-element sets. Then there exists an uncountable closed set $E$ without a copy of any $X \in \mathcal{X}$.

Proof. By Mycielski's Theorem 1 there exists an uncountable, closed set $E$ whose elements are linearly independent over the field $\mathbb{Q}(\cup \mathcal{X})$. Now pick any $X \in \mathcal{X}$ and suppose that there exist real numbers $b$ and $c, c \neq 0$ such that $c \cdot X+b \subseteq E$. For some three different $e_{1}, e_{2}, e_{3} \in E$ we have $c \cdot x_{1}+b=$ $e_{1}, c \cdot x_{2}+b=e_{2}$, and $c \cdot x_{3}+b=e_{3}$. By subtracting these equations from each other we obtain $c\left(x_{1}-x_{2}\right)=e_{1}-e_{2}$ and $c\left(x_{2}-x_{3}\right)=e_{2}-e_{3}$. Now by dividing the first equation by $c\left(x_{1}-x_{2}\right)$, the second by $c\left(x_{2}-x_{3}\right)$ and subtracting we get a linear combination with coefficients from $\mathbb{Q}(\cup \mathcal{X})$,

$$
\frac{1}{c\left(x_{1}-x_{2}\right)} e_{1}-\left(\frac{1}{c\left(x_{1}-x_{2}\right)}+\frac{1}{c\left(x_{2}-x_{3}\right)}\right) e_{2}+\frac{1}{c\left(x_{2}-x_{3}\right)} e_{3}=0
$$

which contradicts the linear independence of $E$.

The above result leads to the question, "Can we have a regular set that would be bigger (of positive Lebesgue measure or of second Baire category) and still not contain a copy of a given three element set?" Here the answer is negative and it follows from Theorem 3 below.

\section{Sets with Copies of Finite Sets}

Throughout the rest of the paper all sets will be subsets of the real line $\mathbb{R}$ unless stated otherwise. $\mu$ will denote Lebesgue measure on $\mathbb{R}$. The following theorem is due to Steinhaus.

Theorem 3 If $X$ is finite and $E$ is of positive Lebesgue measure, then $E$ contains a copy of $X$. 
Proof. Let $\operatorname{card}(X)=n$ and let $E$ be of positive Lebesgue measure. It follows from the Lebesgue Density Theorem (See [8] page 17.) that there exists an interval $[\alpha, \beta]$ such that

$$
\mu(E \cap[\alpha, \beta])>\frac{2 n(\beta-\alpha)}{2 n+1} .
$$

Let $E_{1}=E \cap[\alpha, \beta]$. Without loss of generality let us assume that $X=\left\{x_{1}<\right.$ $\left.x_{2}<\ldots<x_{n}\right\}$ and $x_{1}=0$. Pick $c>0$ small enough to make $c x_{n}<\frac{\beta-\alpha}{2 n+1}$. Since for every $b \in E_{1}-c x_{i}, i=1,2, \ldots, n$ we have $c x_{i}+b \in E_{1} \subseteq E$, it suffices to show that

$$
\bigcap_{i=1}^{n}\left(E_{1}-c x_{i}\right) \neq \emptyset .
$$

To see this, let $J=\left[\alpha-c x_{n}, \beta\right]$ and observe that $E_{1}-c x_{i} \subseteq J$ for every $i=1,2, \ldots n$. Then

$$
\begin{aligned}
\mu\left(\bigcap_{i=1}^{n}\left(E_{1}-c x_{i}\right)\right) & \geq \mu(J)-\sum_{i=1}^{n} \mu\left(J \backslash\left(E_{1}-c x_{i}\right)\right) \\
& \geq(\beta-\alpha)-n\left(\left(\beta-\alpha+c x_{n}\right)-\frac{2 n(\beta-\alpha)}{2 n+1}\right) \\
& \geq(\beta-\alpha)-n\left(\left(\beta-\alpha+\frac{\beta-\alpha}{2 n+1}\right)-\frac{2 n(\beta-\alpha)}{2 n+1}\right) \\
& =(\beta-\alpha)-\frac{2 n(\beta-\alpha)}{2 n+1}=\frac{1}{2 n+1}>0
\end{aligned}
$$

which proves (2) and completes the proof for the $c$ selected above. For any $b \in \bigcap_{i=1}^{n}\left(E_{1}-c x_{i}\right)$ we have $c \cdot X+b \subseteq E$.

The next natural task would be to prove an analogous theorem for sets $E$ with the Baire property. It turns out however that a stronger theorem holds.

\section{Sets with Copies of Countably Infinite Sets}

The following theorem is due to H. I. Miller and P. Xenikakis [6].

Theorem 4 If $X$ is a countable and bounded set and if $E$ is a second Baire category set with the Baire property, then $E$ contains a copy of $X$.

Proof. Proceed as in Theorem 3 except that now we can do better in inequality (1). Namely there exists an interval $[\alpha, \beta]$ such that $[\alpha, \beta] \backslash E$ is meager. (See [8] page 19.) Let $E_{1}=[\alpha, \beta] \cap E$. To simplify the rest of the argument, 
assume that $X \subset \mathbb{R}_{+}$and let $c>0$ be such that $\sup (c \cdot X)<\frac{\alpha-\beta}{2}$. Now it suffices to show that $\bigcap_{x \in X}\left(E_{1}-c x\right) \neq \emptyset$. For $x \in X$ it is easy to see the set $E_{1}-c x$ is residual in $\left[\alpha, \frac{\alpha+\beta}{2}\right]$ and since $X$ is countable, the desired assertion follows. As in Theorem $3 b \in \bigcap_{x \in X}\left(E_{1}-c x\right)$ implies that $c \cdot X+b \subset E$.

It is worth noting (Also see [6].) that a similar proof gives an analogous fact.

Theorem 5 If $X$ is a countable and bounded set and if $E$ is of full measure in some open set $(\mu(V \backslash E)=0$ for some open set $V)$, then $E$ contains a copy of $X$.

Now, if in Theorem 5 we weaken the assumption "of full measure in some open set" to "is of positive measure", then it is no longer true. The strongest result in this direction is due to K. J. Falconer [3].

Theorem 6 Let $\left\{x_{n}\right\}_{n=1}^{\infty}$ be a decreasing sequence converging to zero such that $\lim \inf \frac{x_{n+1}}{x_{n}}=1$. Then there exists a set of positive measure $E$ such that $\left(c \cdot\left\{x_{n}: n=1,2,3, \ldots\right\}+b\right) \backslash E$ is infinite for every number $b$ and every number $c \neq 0$.

Falconer's theorem partially answers an old problem of P. Erdös.

Problem ([2], [5], and also see [4]) Is it true that for every infinite set $X$ there exists a set of positive Lebesgue measure without a copy of $X$ ?

The rest of this note is devoted to the discussion of Erdös' problem. The case of unbounded $X$ is trivial. If $X$ is bounded, then it contains a convergent sequence. Theorem 6 gives a positive answer if $X$ contains a sequence which converges "slower than geometrically". It is worth noting that an intriguing case when $X=\left\{\frac{1}{2^{n}}: n=1,2,3, \ldots\right\}$ remains open (see [4]). Below I would like to present a different partial answer to Erdös' problem. Its proof is quite straightforward (geometric) and well illustrates the nature of the problem. The following lemma converts the Erdös problem to a "tiling puzzle".

Lemma 7 Let $X \subseteq(0,1)$ be a set. The following conditions are equivalent.

1. There exists a set $E, \mu(E)>0$ such that

$$
\forall b \forall c \neq 0\left(c \cdot X+b \cap E^{c} \neq \emptyset\right)
$$

2. There exists a set $E_{1} \subseteq(0,1), \mu\left(E_{1}\right)>0$ such that

$$
\forall 0<c<1\left(E_{1}^{c}+c \cdot X\right)=\mathbb{R}
$$


Proof. Assume 1. Pick an arbitrary $c \neq 0$. Then statement (3) implies that

$$
\begin{gathered}
\forall b \exists y \in E^{c} \exists x \in X(c x+b=y) \\
\forall b \exists y \in E^{c} \exists x \in X(-c x+y=b) \\
\left(-c \cdot X+E^{c}=\mathbb{R}\right)
\end{gathered}
$$

All statements in (5) are equivalent. It is easy to see that $E_{1}=(E+t) \cap(0,1)$, where $t$ is such that $\mu((E+t) \cap(0,1))>0$ satisfies 2 .

Assume 2. By the Lebesgue Density Theorem (See [8] page 17.) there exists a real number $s$ such that $\mu\left(E_{1} \cap\left(-E_{1}+s\right)\right)>0^{2}$. Select $E \subseteq E_{1} \cap\left(-E_{1}+s\right)$ such that $\operatorname{diam}(E)<\operatorname{diam}(X)$. The last inequality gives (3) for $c$ with $|c| \geq 1$. If $-1<c<0$, then (4) yields $\left(E^{c}+(-c) \cdot X=\mathbb{R}\right.$ ) and (5) implies (3) for such $c$. On the other hand if $0<c<1$, then by multiplying the equation $\left(E_{1}^{c}+c \cdot X\right)=$ $\mathbb{R}$ by $(-1)$ and adding $s$ to both sides we obtain $\left(\left(-E_{1}^{c}+s\right)+(-c) \cdot X\right)=\mathbb{R}$. $E^{c}$ contains $\left(-E_{1}^{c}+s\right)$ and as before (5) completes the proof.

Part 2. of Lemma 7 may be viewed as a "puzzle". To better understand this comparison, suppose we are given an infinite set $X \subseteq(0,1)$ and we are looking for a set $E_{1}$ as in the lemma. Recall that every set of positive measure contains a closed set of positive measure. (See [8] page 15.) So we may as well look for a closed set $E_{1}$. This is equivalent to finding its complement, namely an open set $G$ such that

$$
\mu((0,1) \backslash G)>0 \text { and } \forall 0<c<1(G+c \cdot X)=\mathbb{R}
$$

Now $G$ as any open set is a countable union of disjoint open intervals $G_{i}$ (tiles), say $G=\bigcup_{i=1}^{\infty} G_{i}$. Our job is to choose the length and place for each $G_{i}$ in such a way that when each is replaced by the set $\left(G_{i}+c \cdot X\right)$ they will cover the entire numberline. Notice that while the $G_{i}$ do not overlap, the $\left(G_{i}+c \cdot X\right)$ may. Also observe that the whole action takes place between zero and one as we may choose $G$ to include $G_{1}=(-\infty, 0)$ and $G_{2}=(1, \infty)$.

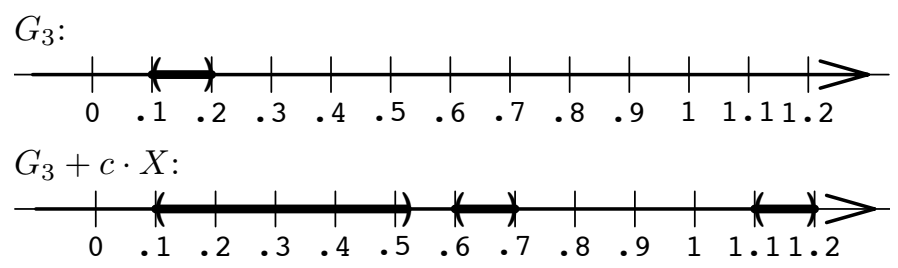

Figure 1: $X=\{2 / n: n=1,2,3, \ldots\}, G_{3}+(.1, .2), c=.5$

\footnotetext{
${ }^{2}-E_{1}=(-1) \cdot E_{1}$
} 
The increasing distances between components of $G_{i}+c \cdot X$ shown in Figure 1 above make them difficult "puzzle pieces". We are going to simplify the problem by using only the biggest connected component $((0.1,0.533 \ldots)$ in Figure 1). To be able to put a finger on its right end, we define $k(\delta)=\min \{k$ : $\left.\forall i \geq k\left(x_{i}-x_{i+1}\right) \leq \delta\right\}$. We also need to account for the variable $c$ and to do so we shall cover $(0,1)$ infinitely many times. In each round the placement of $G_{i}$ 's is designed to work for $c>\frac{1}{n}, n=1,2,3, \ldots$. We shall sometimes write $x(n)$ instead of $x_{n}$.

Theorem 8 Let $X=\left\{x_{n}: n=1,2,3, \ldots\right\} \subseteq(0,1)$ be a decreasing sequence converging to zero. If there exists a sequence $\left\{\delta_{n}\right\}_{n=1}^{\infty}$ such that

$$
\sum_{n=1}^{\infty} \delta_{n}<1 \text { and } \sum_{n=1}^{\infty} x\left(k\left(\delta_{n}\right)\right)=\infty
$$

then there exists a double sequence of intervals $\left\{G_{n m}\right\}_{n, m=1}^{\infty}$ such that $G=$ $\bigcup_{n, m=1}^{\infty} G_{n m}$ has property (6).

Proof. Assume that $\left\{\delta_{n}\right\}_{n=1}^{\infty}$ has property (7). Let $\sigma: \mathbb{N}^{2} \longrightarrow \mathbb{N}$ be a bijection such that

$$
\sum_{m=1}^{\infty} x\left(k\left(\delta_{\sigma(n, m)}\right)\right) \geq n \text { for all } n=1,2,3, \ldots
$$

Now fix an $n$ and define $G_{n m}=\left(a_{n m}, a_{n m}+\delta_{\sigma(n, m)}\right)$ as follows:

$$
\begin{aligned}
a_{n 1} & =0 \\
a_{n m} & =\sum_{i=1}^{m-1}\left[\delta_{\sigma(n, i)}+\frac{1}{n} x\left(k\left(\delta_{\sigma(n, i)}\right)\right)\right]= \\
& =a_{n m-1}+\delta_{\sigma(n, m-1)}+\frac{1}{n} x\left(k\left(\delta_{\sigma(n, m-1)}\right)\right)
\end{aligned}
$$

Since $\mu\left(G_{n m}\right)=\delta_{\sigma(n, m)}$, we have $\mu((0,1) \backslash G) \geq 1-\sum_{n=1}^{\infty} \delta_{n}>0$. To verify the second part of (6) pick a $0<c<1$ and select a natural number $n$ such that $\frac{1}{n}<c$. Due to $(8)$ for any $x \in(0,1]$ we can find an index $m$ such that $a_{n m}<x \leq a_{n m+1}$. It suffices to prove that $G_{n m}+c \cdot X \supseteq\left(a_{n m}, a_{n m+1}\right]$. For all $k \geq k\left(\delta_{\sigma(n, m)}\right)$ the intervals $G_{n m}+c x_{k}$ and $G_{n m}+c x_{k+1}$ overlap. It follows that $G_{n m}+c \cdot X \supseteq\left(a_{n m}, a_{n m}+\delta_{\sigma(n, m)}+c x\left(k\left(\delta_{\sigma(n, m)}\right)\right)\right.$. Since $c>\frac{1}{n}$, we obtain $a_{n m}+\delta_{\sigma(n, m)}+c x\left(k\left(\delta_{\sigma(n, m)}\right)\right)>a_{n m+1}$. 
Corollary 9 If both $\left\{x_{n}\right\}_{n=1}^{\infty}$ and $\left\{\left(x_{n}-x_{n+1}\right)\right\}_{n=1}^{\infty}$ are decreasing sequences converging to zero and $\sum_{n=1}^{\infty} x_{n}=\infty$, then there exists a set of positive measure without a copy of $X=\left\{x_{n}: n=1,2,3, \ldots\right\}$.

Proof. Note that $\delta_{n}=\left(x_{n}-x_{n+1}\right)$ satisfies condition (7). Corollary 9 follows from Theorem 8 and Lemma 7 .

With a more careful choice of $\delta_{n}$ Theorem 8 can be applied to sequences $\left\{x_{n}\right\}_{n=1}^{\infty}$ for which the series $\sum_{n=1}^{\infty} x_{n}$ converges.

Corollary 10 For any real number $\alpha>0$ there exists a set of positive measure without a copy of $X=\left\{\frac{1}{k^{\alpha}}: k=1,2,3, \ldots\right\}$.

Proof. We only need discuss the case of $\alpha>1$. Let $x(k)=k^{-\alpha}$. It turns out that if we let $\delta_{n}=n^{-\frac{\alpha+1}{\alpha}}$, then $x\left(k\left(\delta_{n}\right)\right)$ behaves like $\frac{1}{n}$ and here is why. First we find an upper bound on $k\left(\delta_{n}\right)$. There exists a constant $\xi$ such that

$$
\frac{1}{k^{\alpha}}-\frac{1}{(k+1)^{\alpha}}=\alpha \int_{1 / k+1}^{1 / k} t^{\alpha-1} d t \leq \frac{\alpha}{k^{\alpha-1}}\left(\frac{1}{k}-\frac{1}{k+1}\right) \leq \frac{\xi}{k^{\alpha+1}} .
$$

Now since the inequality $\frac{\xi}{k^{\alpha+1}} \leq n^{-\frac{\alpha+1}{\alpha}}$ implies $k \geq \alpha^{\prime} n^{\frac{1}{\alpha}}$ for some other constant $\alpha^{\prime}$, we obtain $k\left(\delta_{n}\right) \leq\left\lceil\alpha^{\prime} n^{\frac{1}{\alpha}}\right\rceil \leq \beta n^{1 / \alpha}$ for some constant $\beta>0$ and $n>n_{0}$. Therefore

$$
\sum_{n=1}^{\infty} x\left(k\left(\delta_{n}\right)\right)=\sum_{n=1}^{\infty} \frac{1}{k\left(\delta_{n}\right)^{\alpha}} \geq \sum_{n=n_{0}}^{\infty} \frac{1}{\beta^{\alpha} n} .
$$

and the last series is divergent. Hence condition (7) is satisfied.

It should be pointed out that both Corollaries 9 and 10 follow from Falconer's result 6 . Also no sequence $\left\{\delta_{n}\right\}_{n=1}^{\infty}$ satisfies condition (7) if $\left\{x_{n}\right\}_{n=1}^{\infty}$ is a convergent geometric sequence.

Example 1 If $\sum_{n=1}^{\infty} \delta_{n}$ is a convergent series with positive terms and $x(k)=$ $q^{-k}$ for some constant $q>1$, then $\sum_{n=1}^{\infty} x\left(k\left(\delta_{n}\right)\right)$ is also convergent.

Proof. To see this we must first estimate $k\left(\delta_{n}\right)$. By solving the inequality $q^{-k}-q^{-(k+1)} \leq \delta_{n}$ for $k$ we obtain $k \geq \eta-\log _{q} \delta_{n}$ for some constant $\eta$. Thus $k\left(\delta_{n}\right) \geq\lfloor\eta\rfloor+\left\lfloor-\log _{q} \delta_{n}\right\rfloor$ and $x\left(k\left(\delta_{n}\right)\right) \leq q^{-\lfloor\eta\rfloor-\left\lfloor-\log _{q} \delta_{n}\right\rfloor} \leq \eta^{\prime} q^{\log _{q} \delta_{n}}=\eta^{\prime} \delta_{n}$ for some constant $\eta^{\prime}$.

On the other hand there exist sequences $\left\{x_{n}\right\}$ for which Theorem 6 does not apply but Theorem 8 does. 
Example 2 For every sequence $\delta_{n} \searrow 0$ with $\sum \delta_{n}<1$ there exists a sequence $x_{n} \searrow 0$ with property (7) such that $\lim \inf \frac{x_{n+1}}{x_{n}} \leq \frac{1}{2}$.

Proof. Let $\delta_{n} \searrow 0$. By induction we shall define increasing sequences of natural numbers $\left\{s_{n}\right\}_{n=0}^{\infty}$ and $\left\{t_{n}\right\}_{n=0}^{\infty}$ and a decreasing sequence of real numbers $x_{n} \searrow 0$ such that for $n=1,2,3, \ldots$

$$
\begin{gathered}
\frac{x_{t_{n}+1}}{x_{t_{n}}}=\frac{1}{2} \\
k\left(\delta_{s_{n}}\right) \leq t_{n-1}+1 \\
\sum_{i=s_{n-1}+1}^{s_{n}} x\left(k\left(\delta_{i}\right)\right) \geq 1 .
\end{gathered}
$$

Set $s_{0}=t_{0}=0$. To start the induction select an arbitrary $x_{1} \in(0,1)$, let $r_{1}=\left\lceil\frac{1}{x_{1}}\right\rceil$ and $s_{1}=r_{1}$. Now find a natural number $t_{1}$ and a decreasing arithmetic progression $x_{1}, x_{2}, \ldots, x_{t_{1}}$ such that $x_{j}-x_{j+1} \leq \delta_{s_{1}}$ and $\frac{1}{2} x_{t_{1}} \leq \delta_{s_{1}}$. Also set $x_{t_{1}+1}=\frac{1}{2} x_{t_{1}}{ }^{3}$.

Now assume that $s_{n-1}, t_{n-1}$ and $x_{1}, x_{2}, \ldots, x_{t_{n-1}+1}$ are defined and meet conditions (9) (provided $x_{n} \searrow 0$ ). Set $r_{n}=\left\lceil\frac{1}{x_{t_{n-1}+1}}\right\rceil, s_{n}=s_{n-1}+r_{n}$ and find a natural number $t_{n}>t_{n-1}$ with a decreasing arithmetic progression $x_{t_{n-1}+1}, x_{t_{n-1}+2}, \ldots, x_{t_{n}}$ such that $x_{j}-x_{j+1}<\delta_{s_{n}}$ for $j=t_{n-1}+1, t_{n-1}+$ $2, \ldots, t_{n}-1$ and $\frac{1}{2} x_{t_{n}}<\delta_{s_{n}}$. Also set $x_{t_{n}+1}=\frac{1}{2} x_{t_{n}}$. Again conditions (9) can be easily verified.

It should be noted that the above example may be modified to obtain $\liminf \frac{x_{n+1}}{x_{n}}=0$.

We would like to conclude with a remark on arbitrary infinite sets $X$. Let $C$ be a countable set not containing zero. Lemma 7 can be used to show that there exists a set of positive measure not containing any of the sets $c \cdot X+b$ for all $b \in \mathbb{R}$ and all $c \in C$. We deal with each $c$ individually. Observe that the ratio $\mu\left(G_{n}+c \cdot X\right) / \mu\left(G_{n}\right) \rightarrow \infty$ as $\operatorname{diam}\left(G_{n}\right) \rightarrow 0$. So even though $\mu(G)<\infty$, $\sum_{n=1}^{\infty} \mu\left(G_{n}+c \cdot X\right)=\infty$ and we shall have enough "extra length" to cover $(0,1)$ infinitely many times. We leave the details to the reader.

I am grateful to Irek Recław for an interesting discussion.

\footnotetext{
${ }^{3}$ Without having all $x_{n}$ defined it is impossible to determine $x\left(k\left(\delta_{n}\right)\right)$ and verify conditions 9. However, assuming that $x_{n} \searrow 0$ it follows that 9 hold for $n=1$.
} 


\section{References}

[1] J. Arias de Reyna, Some results connected with a problem of Erdös III, Proc. Amer. Math. Soc., 89 (1983), 291-292.

[2] P. Erdös, Problems, Balkanica (Papers presented at the Fifth Balkan Mathematical Congress), 4 (1974), 203-204.

[3] K. J. Falconer, On the problem of Erdös on sequences of measurable sets, Proc. Amer. Math. Soc., 90 (1984), 77-78.

[4] D. H. Fremlin, Problems, (1994) 4.

[5] R. D. Mauldin (editor), The Scottish Book, Birkhauser, Boston, Mass., (1981) 38.

[6] H. I. Miller and P. I. Xenikakis, Some results connected with the problem of Erdös I, Akad. Nauka i Umjet. Bosne i Hercegov. Rad. Odjelj. Prirod. Mat. Nauka, 19 (1980), 71-75.

[7] J. Mycielski, Independent sets in topological algebras, Fund. Math., 55 (1964), 134-141.

[8] J. C. Oxtoby, Measure and Category, Springer-Verlag, New York 1980. 